\title{
Advances in biotechnology and propagation of nut trees in Iran
}

\author{
Kourosh Vahdati*, Mohammad Mehdi Arab, and Saadat Sarikhani \\ Department of Horticulture, College of Aburaihan, University of Tehran, Tehran, Iran
}

\begin{abstract}
As one of the main origin centers of nut trees, Iran is the fourth leading nut crops producer in the world ( $6 \%$ of total nut production). Due to the high genetic diversity, development of new varieties and rootstocks with desirable characteristics have been highly considered by fruit breeders in Iran. In this regard, molecular breeders concentrate on filling the gaps in the conventional breeding with the aim of accelerating breeding programs. Recent advancements in molecular breeding such as next-generation sequencing (NGS) techniques, high-throughput genotyping platforms and genomics-based approaches including genome wide association studies (GWAS), and genomic selection (GS) have opened up new avenues to enhance the efficiency of nut trees breeding. Over the past decades, Iranian nut crops breeders have successfully used advanced molecular and genomic tools such as molecular markers, genetic transformations and high-throughput genotyping to explore the genetic basis of the desired traits and eventually to develop new varieties and rootstocks. Due to a broad international cooperation, a clear perspective is envisaged for the nut breeding programs in Iran, especially based on new biotechnology techniques. The propagation of nut trees in Iran have also been dramatically improved. Different types of grafting and tissue culture (micropropagation or somatic embryogenesis) techniques for propagation of nut crops have been studied intensively in the last 30 years in Iran and the successful techniques have been commercialized. Several certified nurseries are producing grafted and micropropagation plants of walnut, pistachio and other nut crops commercially. A part of the grafted and micropropagaited plants of nut crops in Iran is being exported to the other countries. Establishing modern orchards of nut crops using new cultivars and rootsocks is presently being advised by professional consultants.
\end{abstract}

\section{Introduction}

Central and western Asia (including Iran) are believed to be the origin and domestication center of the nut trees and these ancient species spread to Europe and the US [1,2]. For example, the commercial pistachio varieties were collected from Iran and introduced to the

\footnotetext{
*Corresponding Author: kvahdati@ut.ac.ir
} 
US in 1929 [3]. Also, Iran and Afghanistan are the main center of origin and domestication for vast walnut germplasm then introduced to Eastern Europe, Russia and China [1]. Iran as one of the main origin centers of nut trees, is the fourth leading nut crops producer in the world ( $6 \%$ of total nut production) [4]. Nut trees, especially pistachio, walnut and almond have been widely planted and naturalized in Iran and play a key role in the economy and culture of Iran for both nut and wood production. These trees have been seed-propagated for thousands of year in Iran which have resulted in considerable variation and genetic heterozygosity among the natural populations and accessions $[1,2]$. These valuable native gene pool of nut tree crops populations throughout country have been provided worthy opportunities for nut trees breeders to release new cultivars and rootstocks directly by selection or to use superior genotypes in cross-breeding programs [5].

The nut trees genetic improvement in Iran has rapidly progressed in recent years. In this regard, molecular breeding concentrates on filling the gaps in the conventional breeding with the aim of accelerating breeding programs. In this study, recent advances in nut trees genetic improvement in Iran with the emphasis on molecular breeding as well as their propagation techniques will be discussed.

\section{Molecular marker systems}

Despite the considerable quantity of morphological descriptors for germplasm screening, these methods are costly and time consuming. Therefore, nut trees breeders demand additional and more direct indicators of genetic variability [2]. Information on the nature and degree of genetic diversity of geographically separated nut tree populations could shed light on the distribution of these trees over time $[1,2]$. Also, this genetic information can assist phenotypic evaluation and ultimately will accelerate releasing new cultivars and rootstocks [2].

In the last few decades, the genetic diversity of nut trees in Iran has been extensively studied using various molecular markers systems. Patterns of population structures and genetic diversity of almond have been studied by several molecular marker systems, including isozymes [6], randomly amplified polymorphic DNA (RAPDs) [7], amplified fragment length polymorphism (AFLPs) [8], simple sequence repeat (SSRs) and EST-SSR [9-11]. The use of molecular markers in pistachio breeding and genetic conservation, like in many fruit trees, began with isozymes, then RFLPs, RAPDs, AFLPs, inter simple sequence repeats (ISSRs), sequence-related amplified polymorphism (SRAPs), SSRs, non-coding chloroplast DNA markers and finally single nucleotide polymorphism (SNPs) [2]. Genetic markers systems have been widely used to evaluate walnut genetic diversity in Iran as follow: RAPDs, SSRs, and SCoT markers [1].

Several types of molecular markers have been used for assessment of genetic diversity and structure of nut trees germplasm in Iran. However, there is few studies in the literature on genomic analysis of nut trees genetic resource in Iran using high throughput genotyping platforms. The current advances in next-generation sequencing (NGS) and the continuous decrease in cost have facilitated discovery of single nucleotide polymorphisms (SNPs) across the genome and development of high throughput genotyping technology. Recently, a diversity panel of walnut genotypes from different parts of Iran with a variety of climate zones have been genotyped using the new Axiom J. regia 700K SNP genotyping array and genotyping by sequencing (GBS) [12]. Genomic analysis divided the Iranian walnut panel into four main clusters, based on their geographical regions. Also, a total of 389 accessions of 18 almond species have been genotyped using traditional and new generation DNA markers. The results of this study showed that high resolution melting (HRM) analysis is a suitable approach for future studies in terms of time, cost and efficiency [13]. Considering all the studies mentioned, there is wide areas covered by walnut trees in different parts of 
Iran, which little is known about their genetic diversity and structure so far. Therefore, applying the novel genomic platforms is necessary for uncovering new unexplored genomic diversity in Iranian nut gene pools and accelerating breeding process.

\section{Genome sequencing and association mapping}

Recent remarkable advances in next generation sequencing (NGS) systems and bioinformatics tools have opened up new avenues to use of genomics-based approaches such as genome wide association studies (GWAS), marker assisted selection (MAS), and genomic selection (GS) in nut trees genetic improvement programs [1]. These advancements have the potential to revolutionize the breeding of nut tree crops by reducing the length of the breeding cycle and by improving selection intensity [2]. Accelerated breeding of superior nut tree crops cultivars using genomic-based approaches require reference genome and cost-efficient techniques such as GBS for genome-wide genotyping of large and diverse populations. Recently, the availability of genomic resources is beginning to make possible the use of genomic-assisted breeding in nut tree crops breeding. In pistachio, 93 cultivars and 14 wild $P$. vera genomes and 35 closely related wild Pistacia genomes have been sequenced using Illumina and PacBio sequencing and the results showed genomic admixture occurred among the different wild Pistacia species and key genes for domestication related to tree and seed size [14].

There is a limited research regarding GWAS in nut trees in Iran. The availability of the new Axiom J. regia 700K SNP genotyping array enables walnut breeder to develop genomewide SNP assay with the power to detect genomic regions related to the important traits. Recently, a diverse panel of 95 walnut families (950 seedlings) from Iran with a variety of climate zones genotyped with the new walnut array. GWAS have been performed between phenotypic (nut and water use efficiency related trait), genotypic, and environmental variables and several sets of SNP markers and genes in the genomic regions which is significantly associated with the aforementioned traits was found. These obtained molecular markers can be used in walnut breeding programs in Iran and other countries [15].

\section{Transcriptome and proteome}

Initially, transcriptome analyses in nut tree crops focused on generating ESTs to identify putative candidate genes underling different stages of tree development and abiotic and biotic stresses resistance. High-throughput transcriptome analysis using RNA-Seq approach have been recently incorporated in various nut tree crop research projects. In almond, in the beginning de novo transcriptome assembly and comparative gene expression analysis in response to freezing stress using RNA-Seq data showed significant alterations in 5,112 and 6,926 genes in ovary and anther which were affected by freezing stress. Gene ontology showed differentially expressed genes were involved in metabolic and cellular processes and the expression pattern of eight genes were verified by quantitative real-time PCR (qRT-PCR) [16]. In another study, transcriptome analysis of a frost-tolerant genotype in response to freezing stress using RNA-Seq method revealed 863 upregulated genes and 555 downregulated genes in the frost-treated library. In this study, a highly expressed cold-shock protein was identified which can be used as a marker in future studies [17].

In pistachio, for the first time, RNA-Seq analysis of a pooled sample of different tissues of two pistachio cultivars with contrasting salinity tolerance under control and salt treatment were performed by Illumina Hiseq 2000 platform. In this study, 13,097 transcripts were identified as candidate stress-responsive genes and expression patterns of some of these genes were validated using qRT-PCR. They also identified 11,337 SSRs which can be used 
in pistachio genomic studies in future. Most of the candidate genes identified were involved in plant hormone biosynthesis and signalling and secondary metabolite biosynthesis pathways [18]. Also, the transcriptomic data generated by high throughput sequencing were used for identification of salt-related long noncoding RNAs in Pistacia vera L. They identified 1909 and 1820 salt responsive lncRNAs in Qazvini and Sarakhs as the salt tolerant and the sensitive cultivar, respectively. The gene ontology (GO) analysis showed that identified lncRNAs were involve in the regulation of ATPase, cation transporter, kinase and UDP-glycosyltransferases genes [19]. Recently, RNA segueing of leaf and root samples of pistachio rootstock, $P$. vera L. $c v$. Ohadi, grown under normal and salinity conditions were performed on the Illumina HiSeq X Ten platform. The results of this study showed that jasmonic acid (JA) biosynthetic pathway plays a key role in pistachio salt tolerance [14].

In order to identify candidate genes involved in drought tolerance of walnuts, we sequenced the RNA of walnut leaf ( $c v$. Chandler) grown under normal and water deficit conditions by an Illumina HiSeq 2000 platform. we constructed fifteen different transcriptome assemblies and the results revealed Evidential Gene was the best assembler for the de novo assembly of walnut leaf transcriptome [20].

A limited proteomics and metabolomics studies have been conducted on nut trees in the world and in Iran. Therefore, special attention should be paid to these studies in the future. In pistachio, a recent proteomic analysis was carry out on leaf samples of UCB-1 pistachio rootstock subjected to salinity stress using MALDI-TOF/TOF method [3]. They identified ribonucleoside-diphosphate reductase small chain, polcalcin $\mathrm{Phl} \mathrm{p}$ 7-like and golgin subfamily A member 5 proteins which in previous studies, no role has been reported for them in responding to abiotic stresses in plants [21]. In another study, high-throughput comparative proteomics analysis was conducted on the roots samples of UCB1 and Akbari as tolerance and susceptible pistachio rootstocks, under salinity stress. The results of this study revealed 153 and 340 proteins were upregulated, in UCB1 and Akbari, respectively. The identified proteins were assigned into signal transduction, cell wall and cytoskeleton metabolism proteins groups [1].

\section{Genetic transformation}

Genetic improvement of nut tree crops by conventional breeding is constrained by their long juvenile period, and time-consuming and costly evaluation of agronomic traits in the field. Genetic transformation can accelerate traditional breeding through the transferring of genes (specific traits) into selected genotypes in a short time. To the best of our knowledge, so far, there has been no report of almond and pistachio transformation in Iran. In contrast, agrobacterium mediated transformation of walnut somatic embryos using fld gene were carry out in Dr. Vahdati's lab [22] to improve osmotic stress tolerance. They observed a significant difference between transgenic and non-transgenic plants subjected to salinity stress. Also, the transgenic walnut expressing the fld gene showed higher tolerance to osmotic stress. Walnut somatic embryos transformed with a betaine aldehyde dehydrogenase (badh) gene and additional studies have been done in the same lab [23]. Although with genomic-assisted approaches, breeding periods have been reduced, but still not significantly enough. Thus, applying new genomic editing technologies including zinc finger nucleases (ZFNs), transcription activator-like effector nucleases (TALENs) and clustered regulatory interspaced short palindromic repeat (CRISPR)/Cas-based RNA-guided DNA endonucleases have great potential for promoting the efficiency of nut tree crops breeding in future studies. 


\section{Propagation}

In general, nut trees can be propagated by seeds, cuttings, layering, budding, grafting and micropropagation. Traditionally, nut tree crops have been propagated by seeds and grafting on seedling rootstocks in Iran. Although hardwood and semi-hardwood cuttings can also be used for nut trees propagation, but because of low success rate, they are not commonly used. Like cutting, layering is a low efficiency method for nut trees propagation. Nevertheless, a higher rooting efficiency in stool layering was reported by Vahdati and Khalighi for propagation of Persian walnut. Also, Vahdati et al. reported that low-vigour seedlings rooted more than semi- and high-vigour seedlings in stool layering.

Grafting is one of the common conventional technique for walnut propagation and topworking which is developed in Iran by Vahdati et al. [24]. The different methods and plant materials resulted in different degrees of success under different climatic conditions. Recently, all these methods have been reviewed in detail by Vahdati and Aalifar [25].

Nut trees like other woody plants are still often very difficult to propagate in many different types of propagation. One of the new and important ways of walnut propagation is micropropagation which is a new high-performance technique that has been used successfully to propagate nut trees around the world as well as Iran. Several studies have been conducted to improve the micropropagation of nut trees in Iran [25-27]. In addition, some tissue culture laboratory in Iran are currently propagating nut trees on a large scale by tissue culture technique. In almond, GF677 and NG15 are two of the common rootstock used in high density orchard of Prunus spp., especially for almonds in Iran. Recently, a new culture media for large scale micropropagation of GN15 rootstock was formulated using artificial neural network- genetic algorithm (ANN-GA) [26, 27]. Also, ANNs-GA have been employed to predict optimal in vitro culture medium for Pistacia vera [28]. Fortunately, walnut commercially being propagated by tissue culture methods in Iran by Vahdati. In walnut micropropagation, the acclimatization of micropropagated plants is very difficult because of their susceptibility to diseases and rapid desiccation. In the recent years, many studies have been focused on acclimatization of walnut tissue culture seedlings [36]. Currently, we have acquired the technology necessary to micropropagate some commercial cultivars and rootstocks of nut trees. A part of this technology is currently being transferred to the other countries. Several grafted and micropropagaited plants of nut crops in Iran are being exported to the other countries. Establishing modern orchards of nut crops using new cultivars and rootsocks is currently being consulted by professional advisers.

\section{References}

1. K. Vahdati, M. M. Arab, S. Sarikhani, M. Sadat-Hosseini, C. A. Leslie, P. J. Brown, Advances in Plant Breeding Strategies: Nut and Beverage Crops, 401-472 (2019) https://link.springer.com/chapter/10.1007/978-3-030-23112-5_11

2. A. Sheikhi, M. M. Arab, P. J. Brown, L. Ferguson, M. Akbari, Advances in Plant Breeding Strategies: Nut and Beverage Crops, 353-400, (2019) https://link.springer.com/chapter/10.1007/978-3-030-23112-5_10

3. D. Geisseler, W. R. Horwath, California Department of Food and Agriculture Fertilizer Research and Education Program (FREP), 1-14 (2016) https://www.cdfa.ca.gov/is/docs/15-0231-Geisseler.pdf

4. INC. International Nut and Dried Fruits Press (2020) https://www.nutfruit.org/industry/press-room

5. A. Ebrahimi, R. Fatahi, Z. Zamani, Sci Hortic 130(1), 146-151 (2011) https://www.sciencedirect.com/science/article/pii/S0304423811003189 
6. A. Vezvaei, InXXVI International Horticultural Congress: Genetics and Breeding of Tree Fruits and Nuts, 622, 451-456 (2002) https://www .actahort.org/books/622/622_47.htm

7. K. Nikoumanesh, A. Ebadi, M. Zeinalabedini, Y. Gogorcena, Sci. Hortic 129(1), 108118 (2011) https://www.sciencedirect.com/science/article/pii/S0304423811001257

8. M. Zeinalabedini, J. Dezhampour, P. Majidian, M. Khakzad, B. M. Zanjani, A. Soleimani, M. Farsi, Sci. Hortic, 172, 258-264 (2014) https://www.sciencedirect.com/science/article/pii/S0304423814002003

9. M. Zeinalabedini, S. Sohrabi, K. Nikoumanesh, A. Imani, M. Mardi, Sci. Hortic. 298(10), 1917-1929 (2012) https://link.springer.com/article/10.1007/s00606-012-0691-8

10. M. Zeinalabedini, P. Majidian, R. Ashori, A. Gholaminejad, M.A. Ebrahimi, P. MartinezGomez, Sci. Hortic, 247, 49-57 https://www.sciencedirect.com/science/article/pii/S0304423818308744

11. A. Rahemi, R. Fatahi, A. Ebadi, T. Taghavi, D. Hassani, T. Gradziel, K. Folta, J. $\begin{array}{lllll}\text { Chaparro, Plant } & \text { Syst. } & \text { Evol., } & \text { 298, } & 173-192\end{array}$ https://link.springer.com/article/10.1007/s00606-011-0536-X

12. M.M. Arab, A. Marrano, R. Abdollahi-Arpanahi, C.A. Leslie, H. Askari, D.B. Neale, K. Vahdati, Sci. Rep., 9, 6376 (2019) https://www.nature.com/articles/s41598-019-42940-1

13. K. Sorkheh, M. Koohi-Dehkordi, S. Ercisli, A. Hegedus, J. Halász, Sci. Rep., 7, 5966 (2017) https://www.nature.com/articles/s41598-017-06084-4

14.L. Zeng, X.L. Tu, H. Dai, F.M. H, B.S. L, M.S. Wang, H. Asadollahpour-Nanaei, A. Tajabadipour, M. Mansouri, X.L. Li, L.L. Ji, D. M. Irwin, H. Zhou, M. Liu, H.K Zheng, A. Esmailizadeh, D.D. Wu, Genome Biol, 20, 1 (2019). DOI: 10.1186/s13059-019-16863

15. M. M. Arab, A. Marrano, R. Abdollahi-Arpanahi, C. A. Leslie, H. Cheng, D. B. Neale, K. Vahdati, J. Exp. Bot. 71, 3 (2020). DOI: 10.1093/jxb/erz467

16. S. Mousavi., A. Alisoltani., B. Shiran1, H. Fallahi, E. Ebrahimie, A. Imani, S. Houshmand, PloS One. 9, 8 (2014). doi:10.1371/journal.pone.0104541

17. B. Hosseinpour, S. Sepahvand, K. K. Aliabad, M. R. Bakhtiarizadeh, A. Imani, R. Assareh, S.A. Salami, Mol. Genet. Genomics. 293, 1 (2018). https://doi.org/10.1007/s00438-017-1371-8

18. M. M.Jazi, S. M. Seyedi, E. Ebrahimie, M. Ebrahimi, G. De Moro, C. Botanga, BMC Genomics 18, 1 (2017). https://doi.org/10.1186/s12864-017-3989-7

19. M. Jannesar, S. M. Seyedi, M. M.Jazi, V. Niknam, H. Ebrahimzadeh, C. Botanga, Sci. Rep. 10, 1 (2020). https://doi.org/10.1038/s41598-020-62108-6

20. M. Sadat-Hosseini, M.R. Bakhtiarizadeh, N. Boroomand, M. Tohidfar, K. Vahdati, PloS One. 15, 4 (2020). https://doi.org/10.1371/journal.pone.0232005

21. K. J. Goharrizi, A. Baghizadeh, M. Kalantar, F. Fatehi, J. Plant Growth Regul. 15 (2019). https://doi.org/10.1007/s00344-019-10004-3

22. M. A. Sheikh Beig Goharrizi, A. Dejahang, M. Tohidfar, A. Izadi Darbandi, N. Jose Carrillo, M. R. Hajirezaei, K. Vahdati, J. Agr. Sci. Tech.-Iran 18, 2 (2016). https://jast.modares.ac.ir/article-23-5369-en.pdf

23.F. Rezaei Qusheh Bolagh, A. Solouki, M. Tohidfar, M. Zare Mehrjerdi, K. Vahdati. J. Hortic. Sci. Biotech. (2020). https://doi.org/10.1080/14620316.2020.1812446

24. K. Vahdati, D. Hassani, R. Rezaee, M. H. Jafari Seyedi, S. Sarikhani Khorami. In: Following walnut footprints (Juglans regia L.) cultivation and culture, folklore and history, traditions and uses. Scripta Hortic. 17 (2014). https://www.ishs.org/scriptahorticulturae/following-walnut-footprints-juglans-regia-l-cultivation-and-culturefolklore

25. K. Vahdati, M. Aalifar, Acta Hortic. 16 (2015). 10.17660/ActaHortic 2016.1139 .80

26. M. M. Arab, A. Yadollahi, A. Shojaeiyan, H. Ahmadi, Front. Plant Sci. 7, 1526 (2016). doi: 10.3389/fpls.2016.01526 
27. M. M Arab, A. Yadollahi, M. Eftekhari, H. Ahmadi, M. Akbari, S. Sarikhani-Khorami, Sci. Rep. 8(1), 1-18 (2018). https://doi.org/10.1038/s41598-018-27858-4

28. E. Nezami-Alanagh, G. Garoosi, S. Maleki, M. Landín, P. Pablo Gallego, Plant Cell Tiss. Organ Cult. 129, 19-33 (2017). https://doi.org/10.1007/s11240-016-1152-9

29.Z. M. Asayesh, K. Vahdati, S. Aliniaeifard, Sci. Hortic. 224, 1-7 (2017). https://doi.org/10.1016/j.scienta.2017.04.023 